\title{
Fourth-order Gauss Curvature Driven Diffusion for Image Denoising
}

\author{
P. Jidesh and Santhosh George
}

\begin{abstract}
Second-order curvature driven diffusion methods such as mean curvature driven diffusion and Gauss curvature driven diffusion were introduced in the literature for image denoising. However, the second-order Partial Differential Equations modify smooth areas in images into piece-wise constant areas resulting in a staircase effect. Moreover, important information present in images such as textures is often lost in the course of evolution. In this paper, we propose a fourth-order diffusion equation, which uses a function of Gauss curvature as the conductance term to control the magnitude of diffusion. The fourth-order PDE helps to preserve the planar approximation by approximating the observed image with a piecewise planar image. This provides a more natural look to the filtered image. The Gauss curvature based conductance term preserves curvy edges, ramps and corners, with non-zero curvature value. The visual and quantitative results of the method are presented and compared with some of the existing PDE based denoising methods.
\end{abstract}

Index Terms-Fourth-order PDE, gauss curvature, denoising, edge preserving.

\section{INTRODUCTION}

Image denoising is one of the fundamental problems in image processing and computer vision. The image denoising problem is to reconstruct the original image $u(x, y)$ from a noisy observed image $u_{0}(x, y)$. The image degradation can be mathematically modeled as:

$$
u_{0}(x, y)=u(x, y)+\eta(x, y)
$$

where $u_{0}(x, y)$ is the observed image defined on $\Omega$ and $\Omega \subset R^{2}$ denotes an open bounded set with Lipschitz boundary, $u(x, y)$ is the actual image and $\eta$ is an additive white Gaussian noise with zero mean and variance $\sigma^{2}$. However, the image denoising problem is ill-posed in the sense of Hadamard [1].

Partial differential equation (PDE)-based methods have been widely used for image denoising over the last two decades. These PDEs are either formulated directly or are derived from a variational energy minimization problem. They include Total Variation denoising [2] and Non-Linear Diffusion models [3] and [4]. Eventhough these methods preserve edges well, the images resulting from the application of these techniques suffer from staircase effect where the smooth regions are converted into piecewise

Manuscript received February 22, 2012; revised April 23, 2012.

The authors are with National Institute of Technology, Karnataka, and Mangalore, INDIA-575025 (e-mail: jidesh@nitk.ac.in, sgeorge@nitk.ac.in). constant areas. Furthermore, these second-order filters are not so efficient in preserving textures and finer scale details in the image.

An effective solution to these problems has been introduced by You and Kaveh [4]. In [4] a fourth-order PDE is used for noise removal. A significant reduction of staircase effect and a much better preservation of textures are achieved by this method. However, the fourth-order diffusion methods can result in edge distortion, since they dampens high frequency components much faster than the second-order diffusion.

Another second-order anisotropic method which denoises images considerably is the Mean Curvature Motion (MCM), introduced in image processing by Marquina and Osher [5]. In MCM the level-curves in the image are evolved with a speed proportional to their mean curvature at any point. But many important structures, which have nonzero mean curvature values, are badly affected by MCM. For example, curvy edges and corners with nonzero mean curvature values are blurred by the mean curvature flow.

This paper is organized as follows. In Section 2, we briefly review PDE based methods for image denoising. In Section 3 we propose our method and explain the denoising features of the proposed PDE. Section 4 explains the numerical implementation we have employed for solving the PDE. Section 5 is devoted to the numerical experiments performed using the proposed model with visual and quantitative results. We conclude the paper in Section 6.

\section{PDE BASED DENOISING}

Though PDE's are used extensively for image denoising one of the remarkable approach of using non-linear PDE for image denoising was suggested by Perona and Malik[3].They named this model as anisotropic diffusion model in [3], and it is defined as:

$$
\frac{\partial u}{\partial t}=\nabla \cdot(g(\|\nabla u\|) \nabla u)
$$

where $g($.$) is the diffusivity function which is a$ non-increasing function of the magnitude of the image gradient and $u(x, y, t)$ is the image function evolving in time $t$. The symbols " $\nabla . "$ and $\|$.$\| are used for mathematical$ notations divergence and Euclidean norm $\left(\sqrt{\left(u_{x}^{2}+u_{y}^{2}\right)}\right)$, respectively. Here $u_{x}$ and $u_{y}$ denotes the gradient along 
$x$ and $y$ directions, respectively. We assume homogenous Neumann boundary conditions for all the PDE's defined in this paper, that is: $\left(\frac{\partial u}{\partial \vec{n}}=0\right)$, where $u$ is the image function and $\vec{n}$ is the outward normal and the initial condition $u(x, y, 0)=u_{0}(x, y)$, where $u_{0}(x, y)$ is the initial image. The diffusivity function avoids smoothing across edges and allows greater diffusion in smooth areas. Detailed analysis of Perona-Malik equation indicates that, when there is no backward diffusion, a level image is the only solution to the problem, so anisotropic diffusion will evolve toward the formation of a level image function. Since anisotropic diffusion is designed such that, smooth areas are diffused faster than less smooth ones, the blocky effects will appear in the early stage of diffusion, even though all the blocks will finally merge to form a level image. When there is backward diffusion, however, any step image (piecewise level image) is a global minimum of the energy functional, so blocks will appear in the early stage of the diffusion and will remain as such. So the solution of this PDE leads to a piecewise constant approximation of $u$. This PDE may even cause the enhancement of the image if the parameters are not fine-tuned, resulting in unnatural images. Moreover, the problem is not well-posed mathematically. Therefore, the existence of a unique solution cannot be proved. Perona Malik diffusion does not work well when applied to high noisy images, because the noise introduces important oscillations of the gradient [6].

Higher order PDE's are introduced in the literature to better approximate the image by a piecewise planar image instead of a piecewise constant image. The first work in this direction was done by You and Kaveh [4]. In their work, they proposed a fourth-order PDE based method for noise removal. They called this a Fourth-order Diffusion model (FOD). This method seeks to minimize a cost functional, which is an increasing function of the absolute value of the Laplacian of the image intensity function. Since the Laplacian of an image at a pixel is zero if the image is planar in its neighbourhood, these PDEs attempt to remove noise and preserve edges by approximating an observed image with a piecewise planar image. The fourth-order method defines the following functional in the space of continuous image over the area of support $\Omega$ :

$$
E(u)=\int_{\Omega} f\left(\left|\nabla^{2} u\right|\right) d x d y,
$$

where $\nabla^{2} u$ is the Laplacian of the image function $u(x, y)$, |.| denotes the absolute value function and $f($.$) is a strictly$ increasing function. The Euler equation associated with this functional can be written as:

$$
\nabla^{2}\left[f^{\prime}\left(\left|\nabla^{2} u\right|\right) \nabla^{2} u\right]=0
$$

where $f^{\prime}$ is the first order differential of the function $f$. The solution to this equation can be obtained by following the gradient descent procedure:

$$
\begin{aligned}
& \frac{\partial u}{\partial t}=-\nabla^{2}\left[f^{\prime}\left(\left|\nabla^{2} u\right|\right) \frac{\nabla^{2} u}{\left|\nabla^{2} u\right|}\right] \\
& =-\nabla^{2}\left[c\left(\left|\nabla^{2} u\right|\right) \nabla^{2} u\right] \\
& u(x, y, 0)=u_{0}(x, y) .
\end{aligned}
$$

It is proved by You and Kaveh that the planar images are the only minimum of $E(u)$ if $f($.$) is a convex function or$ equivalently if

$$
f^{\prime \prime}(s) \geq 0, \forall s \geq 0 \text {. }
$$

Hence if the cost functional is convex, the evolution of the PDE in (5) will eventually results in smoothing the image until it becomes a complete planar image. And it is further shown that even if the functional $E(u)$ is non-convex, local/global minima may exist for the functional and piecewise planar images are such minima. Apart from this the method of You and Kaveh preserves textures in the image well when compared to the second-order methods defined in the literature.

The mean curvature motion introduced by Marquina and Osher[5] diffuses anisotropically at a faster rate. Here each level-curve of the initial image is evolved in the direction normal to itself with a speed equal to their mean curvature. The PDE associated with this method is:

$$
\frac{\partial u}{\partial t}=\|\nabla u\| \nabla \cdot\left(\frac{\nabla u}{\|\nabla u\|}\right)
$$

where $\nabla u(x, y)$ is the gradient image. The mean curvature represents a degenerate diffusion term which diffuses in the direction orthogonal to its gradient $\nabla u$ and does not diffuse at all, in the direction of $\nabla u$. However, MCM has the tendency to make the curved edges curvier during the evolution and the edges become blurred when a reasonable denoising is attained.

On the other hand there has been considerable interest in geometry driven image diffusion. The mean curvature has been used as a conductance term in the diffusion equation by T.F.Chan et al. in [7], which is named as Mean Curvature Driven Diffusion (MCDD). The diffusion equation is:

$$
\frac{\partial u}{\partial t}=\nabla \cdot(\phi(M) \nabla u)
$$

where

$$
M=\frac{u_{x}^{2} u_{y y}-2 u_{x} u_{y} u_{x y}+u_{y}^{2} u_{x x}}{1+u_{x}^{2}+u_{y}^{2}}
$$


Is the mean curvature. In [7], mean curvature based diffusion is used for image inpaiting. The amount of diffusion is determined by the mean curvature at a point, i.e. the diffusion gets stronger when the isophotes are having a larger curvature value, while it dies away as the isophotes stretch out.

Even though it is not proposed earlier in the literature, it is indeed possible to use the fourth-order diffusion with mean curvature as the conductance function, for denoising images. This takes advantage of the texture preserving capabilities of fourth-order diffusion and noise removing capability of the mean curvature driven diffusion. The forth-order mean curvature driven diffusion can be formulated as below:

$$
\frac{\partial u}{\partial t}=-\nabla^{2}\left(\phi(M) \nabla^{2} u\right)
$$

where $M$ is given in (9). Even though the Mean curvature driven fourth-order diffusion works better than the ordinary fourth-order diffusion and mean curvature driven second-order diffusion, the curvy edges, ramps and corners are not perfectly retained by this diffusion method.

Instead of mean curvature Lee and Seo [8] proposed to use a function of Gauss curvature (GCDD) as the diffusion coefficient in (2) which is modeled by:

$$
\frac{\partial u}{\partial t}=\nabla \cdot(\phi(G) \nabla u)
$$

where $G$ is the Gauss curvature of the surface $z=u(x, y)$. The function $\phi$ is chosen as a monotonically increasing function of the absolute value of the Gauss curvature. The conductance function $\phi$ is dependent on the value of the Gauss curvature at the local region and hence controls the amount of diffusion. The diffusion is large in regions where Gauss curvature has large value and will not diffuse when it is zero. As the noise has a large Gauss curvature value it is effectively removed by Gauss Curvature Driven Diffusion (GCDD). Even though GCDD is able to preserve curvy edges and ramps much better than mean curvature motion, they both share the disadvantage of forming piecewise constant images and removal of texture information.

\section{PROPOSED METHOD}

We propose a fourth-order diffusion equation with Gauss curvature as the conductance term for noise removal, mathematically it is:

$$
\frac{\partial u}{\partial t}=-\nabla^{2}\left(\phi(G) \nabla^{2} u(x, y)\right)
$$

where $\nabla^{2} u(x, y)$ is the Laplacian of the image function $u(x, y)$ and

$$
G=\frac{u_{x x} u_{y y}-u_{x y}^{2}}{\left(1+u_{x}^{2}+u_{y}^{2}\right)^{2}},
$$

is the Gauss curvature at the point $(x, y)$. The function $\phi$ is chosen as a monotonically increasing function of the absolute value of the Gauss curvature. Since the rate of convergence is very slow in case of GCDD if we take $\phi()=.|$.$| , as coined$ out in the work [8]. Therefore, here we propose to use a function which will result in faster convergence, the function can be defined as:

$$
\phi(x)=\frac{|x|}{\max (|x(i, j)|), \forall_{i, j}},
$$

With this choice the diffusion gets stronger in areas where the isophotes are having large Gauss curvature value and the diffusion will be less in areas having low Gauss curvature value. The motivation behind the proposed PDE is to better denoise smooth areas without staircase effect and to preserve textures and edges well. This is done by including Gauss curvature as the conductance term in a fourth-order PDE diffusion equation. The fourth-order PDE is able to preserve more textures and they minimize the block effect while denoising images, where as the Gauss curvature helps in retaining structures, with non-zero mean curvature values.

There are a number of advantages when using Gauss curvature as the diffusion coefficient when compared to mean curvature. The mean curvature is defined as the sum of principal curvatures. The Gauss curvature is defined as the product of principal curvatures and when any of the principal curvatures is zero the Gauss curvature value will be zero. Thus the Gauss curvature in a region may be zero even if the mean curvature is not. Therefore, structures which are not preserved by mean curvature flow can be preserved by the Gauss curvature flow. For example, a sharp edge is not preserved by mean curvature flow since a sharp edge has a very large maximum principal curvature across the edge. However it is preserved by Gauss curvature flow as the minimum principal curvature along the edge is zero. Curvy edges or radial functions are preserved by Gauss curvature flow, since the curvature in the direction perpendicular to the tangent of the level curve is zero, but they are not preserved by mean curvature flow. Image structures that have low gradient magnitudes are not preserved successfully by other gradient based noise-removal schemes, but they can be preserved by the Gauss curvature flow, if the Gauss curvature is zero at such structures. The noise, which has a large Gauss curvature value, is effectively removed by the Gauss curvature flow. These properties of Gauss curvature are combined with the texture preserving capabilities of fourth-order PDEs to obtain a good quality reconstructed image in our algorithm. Furthermore, the fourth-order PDEs help to denoise the image better, providing a more natural appearance to the filtered image, without causing the staircase artifacts.

\section{NUMERICAL IMPLEMENTATION}

The explicit scheme is used for numerical computation, and the evolution is based on the following iterations:

$$
u^{n+1}=u^{n}-\Delta t\left(\nabla^{2}\left(\phi(G) \nabla^{2} u\right)\right) \text {, }
$$




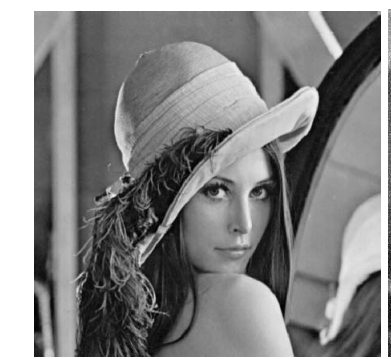

(a)

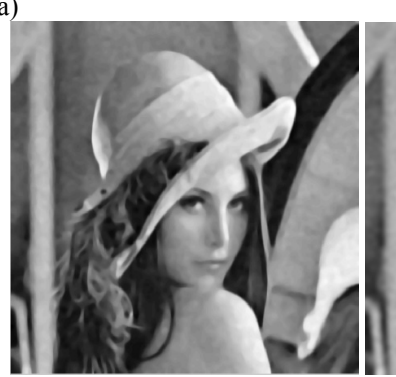

(d)

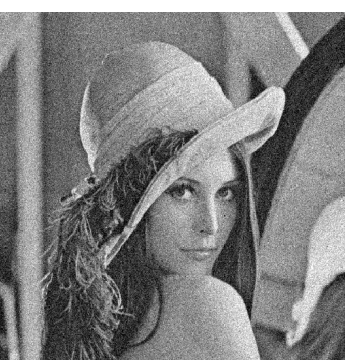

(b)

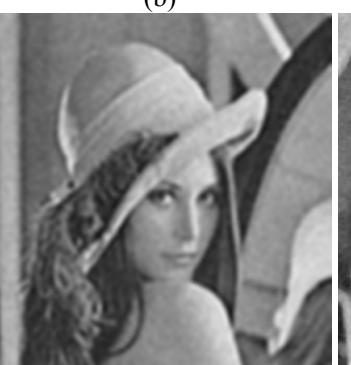

(e)

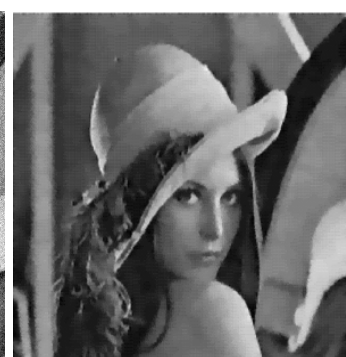

(c)

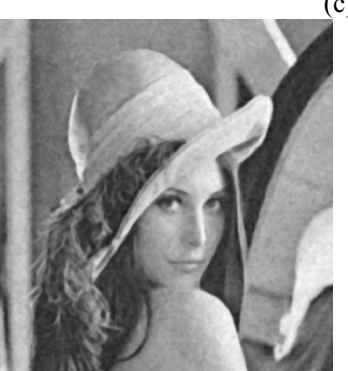

(f)

Fig. 1. Starting from left top: (a) The original image (b) noisy image with Gaussian noise 6.5dB (c) Image denoised with GCDD (d) Image denoised with MCM (e) Image denoised with Forth-order diffusion (f) Image denoised with the proposed method.

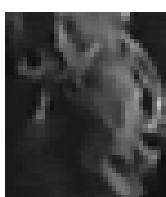

(a)

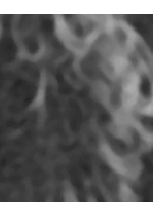

(b)

Fig. 2. The enlarged part of the textured area in the image (a) with GCDD (b) with the proposed method

where $\Delta t$ is the time step and $\phi(G)$ denotes the conductance term (that controls the amount of diffusion) based on Gauss curvature $G$ at the given point, $u$ is the image function, $u^{n}$ and $u^{n+1}$ are the image functions in the $n^{\text {th }}$ and $n+1^{\text {th }}$ iterations respectively. The discretization of the differentiation $-\nabla^{2}\left(\phi(G) \nabla^{2} u(x, y)\right)$ is given in (16). The value of $\phi(x)$ is calculated using (14), in order to get a faster convergence. Note that,

$$
\begin{aligned}
& -\nabla^{2}\left(\phi(G) \nabla^{2} u(x, y)\right) \\
& =4 \phi_{i, j}(G)\left[u_{i+1, j}+u_{i-1, j}+u_{i, j+1}+u_{i, j-1}-4 u_{i, j}\right] \\
& -\phi_{i+1, j}(G)\left[u_{i+2, j}+u_{i, j}+u_{i+1, j+1}+u_{i+1, j-1}-4 u_{i+1, j}\right] \\
& -\phi_{i-1, j}(G)\left[u_{i-2, j}+u_{i, j}+u_{i-1, j+1}+u_{i-1, j-1}-4 u_{i-1, j}\right], \\
& -\phi_{i, j+1}(G)\left[u_{i, j+2}+u_{i, j}+u_{i+1, j+1}+u_{i-1, j+1}-4 u_{i, j+1}\right] \\
& -\phi_{i, j-1}(G)\left[u_{i, j-2}+u_{i, j}+u_{i+1, j-1}+u_{i-1, j-1}-4 u_{i, j-1}\right]
\end{aligned}
$$

where $\phi(i+1, j+1)$ is the value of function $\phi$ at $(i+1, j+1)^{\text {th }}$ location. Here we use the central difference scheme to get the discretization and the space scale $h$ is taken as 1 .

\section{RESUlTS AND DisCUSSIONS}

We tested all the methods choosing the following parameters: $\Delta t=0.01, h=1$, the Gaussian additive noise with mean 0 and standard deviation $\sigma_{G}^{2}=5$, was added to the image. All the images are of resolution 512X512 pixels. In Fig. 1 the result of each of the method discussed in the previous sections are displayed. And the comparative result in terms of their noise removing capacity is listed out in the Table I with Signal to Noise Ratio(SNR) values.

TABLE I: THE SNR OF VARIOUS METHODS COMPARED WITH THE PROPOSED ONE

\begin{tabular}{|l|l|l|l|l|l|}
\hline \multirow{2}{*}{ Image } & \multicolumn{5}{|c|}{ SNR of noisy and processed images } \\
\cline { 2 - 6 } & $\begin{array}{l}\text { Nois } \\
\boldsymbol{y}\end{array}$ & $\boldsymbol{M C M}$ & $\begin{array}{l}\text { FO } \\
\boldsymbol{D}\end{array}$ & GCDD & $\begin{array}{c}\text { The } \\
\text { Proposed } \\
\text { Method }\end{array}$ \\
\hline Lena & 6.8 & 12.7 & 10.8 & 12.9 & 13.5 \\
\hline Barbara & 6.8 & 12.8 & 11.2 & 13.1 & 14.0 \\
\hline Toys & 6.8 & 13.0 & 10.6 & 13.1 & 13.1 \\
\hline
\end{tabular}

The proposed method yields better quality images when compared to the other methods as evident from Fig. 1. Even though the MCM removes the noises it will blur-out most of the curvy edges corners and ramps. The GCDD is able to preserve these edges penalizing the texture details present in the image. The proposed method preserves the curvy edges and corners without penalizing the texture contents of the image. The SNR of noisy image and image denoised with various methods are listed in Table I. It is quite evident that the SNR of the proposed method is better when the images are having more textures and it is comparable to GCDD when the images are smooth or less textured. It is clear from Fig. 1, that the proposed method retains the textures much better as compared to the GCDD. Fig. 2 shows an enlarged portion of highly textured area in the image and one can observe that the proposed method retains the textures where as GCDD 
smooth out the textures. So the fusion of fourth-order and Gauss curvature characteristics make the proposed method a better choice.

\section{CONCLUSIONS}

In this paper we have proposed implemented and tested a method that combines the features of fourth-order diffusion and Gauss curvature. This method substantially denoises the images without penalizing much on the textures, curvy edges, ramps, and corners. The results provided satisfactorily endorse the capability of the method to denoise the images preserving the details and making the images appear quite natural.

\section{REFERENCES}

[1] J. Hardmard, "Lecturer on Cauchy's, problem in linear partial differential equations," New York: Dover publication, 1953.

[2] L. Rudin, S. Osher, and E. Fatemi, "Nonlinear Total variation based Noise Removal Algorithms," Physica D, vol. 60, no. 1, pp. 259-268, 1992.

[3] P. Perona and J. Malik, "Scale-Space and edge detection using anisotropic diffusion," IEEE Transactions on Pattern Analysis and Machine Intelligence, vol. 12, no. 7, pp. 629-639, 1990.

[4] Y.L. You, and M, Kaveh, "Fourth-Order Partial Differential Equation for Noise Removal," IEEE Transactions on Image Processing, vol. 9 , no. 10, pp. 1723-1730, 2000.

[5] A. Marquiana and S. Osher, "Explicit algorithms for a new time-dependant model based on level set motion for non linea deblurring and noise removal," SIAM J.Sci. Comput, vol. 22, no. 2, pp. 387-405, 2000.

[6] Z. C. Lin and Q. Y. Shi. "An anisotropic diffusion PDE for noise reduction and thin edge preservation," in Proceedings of the International Conference on Image Analysis and Processing, pp. 102-107, 1999.

[7] T. F. Chan, S. Kang, and J. Shen, "Euler's elastica and curvature-based image inpainting," SIAM J. Appl. Math., vol. 63, no. 2, pp. 564-592, 2002.

[8] S. H. Lee and J. H. Seo, "Noise removal with Gauss curvature-driven diffusion", IEEE Trans. Image Process, vol. 14, no. 7, pp. 904-909, 2005.

P. Jidesh received his MCA from National Institute of Technology, Calicut, India and M.Tech in Computer Science from University of Kerala, India. He is currently working as an Assistant Professor in the Department of Mathematical and Computational Sciences, National Institute of Technology Karnataka (NITK), India. He has guided many M.Tech dissertations and MCA projects. He has published papers in international journals and proceedings of international conferences in the area of image and signal restoration and enhancement. His research interests include PDE and variational methods in image processing.

Santhosh George received his Ph.D in Mathematics from Goa University. $\mathrm{He}$ is currently working as an Associate Professor in the Department of Mathematical and Computational Sciences, National Institute of Technology Karnataka, India. He has published several papers in international journals of good repute and proceedings of the international conferences. His research interests include functional analysis, inverse and ill-posed problems and its applications. He has supervised many M.Tech dissertations. Two students were awarded Ph.D. degree under his supervision. Currently three Ph.D scholars are working under his supervision. 